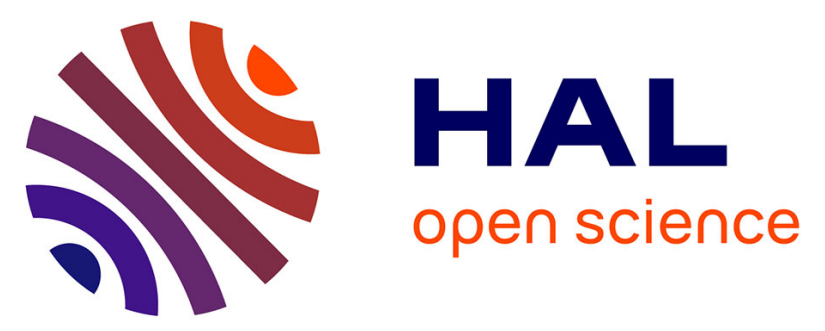

\title{
Quantification of the electrostatic and covalent interaction between whey proteins and low methoxyl pectin using PFG-NMR diffusometry
}

Arima Diah Setiowati, Lien Vermeir, Lorenz de Neve, Ali Sedaghat Doost, Davy Sinnaeve, Paul van Der Meeren

\section{To cite this version:}

Arima Diah Setiowati, Lien Vermeir, Lorenz de Neve, Ali Sedaghat Doost, Davy Sinnaeve, et al.. Quantification of the electrostatic and covalent interaction between whey proteins and low methoxyl pectin using PFG-NMR diffusometry. Magnetic Resonance in Chemistry, 2019, Latest Developments and Applications of magnetic resonance in food science, 57 (9), pp.719-729. 10.1002/mrc.4812 . hal02538690

\section{HAL Id: hal-02538690 \\ https://hal.science/hal-02538690}

Submitted on 20 Apr 2020

HAL is a multi-disciplinary open access archive for the deposit and dissemination of scientific research documents, whether they are published or not. The documents may come from teaching and research institutions in France or abroad, or from public or private research centers.
L'archive ouverte pluridisciplinaire HAL, est destinée au dépôt et à la diffusion de documents scientifiques de niveau recherche, publiés ou non, émanant des établissements d'enseignement et de recherche français ou étrangers, des laboratoires publics ou privés. 


\title{
Quantification of the electrostatic and covalent interaction between whey proteins and low methoxyl pectin using pfg-NMR diffusometry
}

\author{
Arima Diah Setiowati ${ }^{1}$, Lien Vermeir ${ }^{1}$, Lorenz De Neve ${ }^{l}$, Ali Sedaghat Doost ${ }^{1}$, Davy \\ Sinnaeve ${ }^{2}$, Paul Van der Meeren ${ }^{1}$ \\ ${ }^{1}$ Particle and Interfacial Technology group, Department of Green Chemistry and \\ Technology, Faculty of Bioscience Engineering, Ghent University, Coupure Links 653, 9000, \\ Gent, Belgium \\ ${ }^{2} N M R$ and Structure Analysis Unit, Department of Organic and Macromolecular Chemistry, \\ Faculty of Sciences, Ghent University, Campus Sterre, S4, Krijgslaan 281, B-9000 Ghent, \\ Belgium
}

\section{Introduction}

Nowadays, complexes of proteins and polysaccharides receive a lot of attention due to their high functionality. This type of complexes can be applied in a wide range of products, such as in beverages ${ }^{[1,2]}$, in gels ${ }^{[3]}$, or in microencapsulation ${ }^{[4,5]}$. These complexes can be formed either through electrostatic interaction or covalent bonds. Electrostatic interaction involves modification of the charge properties of the biopolymers to facilitate the formation of the complexes and is influenced by different factors such as $\mathrm{pH}$, ionic strength, as well as charge density of the protein and polysaccharides ${ }^{[6]}$. There are several studies on electrostatic interaction between proteins and polysaccharides and its application in emulsions ${ }^{[7-9]}$. On the other hand, covalently bound protein-polysaccharide conjugates are normally obtained via Maillard type reactions ${ }^{[2,10,11]}$. This type of conjugates is obtained by exposing a mixture of proteins and polysaccharides to elevated temperatures at certain relative humidity for a certain period of time ${ }^{[12]}$. Previous studies have shown that protein-polysaccharide conjugates have a better functionality than protein/polysaccharides alone ${ }^{[2,13,14]}$. It has been reported that conjugation of WPI and LMP increases the heat stability of WPI as well as its emulsifying activity $[13,15,16]$.

pfg-NMR has been used frequently to measure the diffusion coefficient of different materials and chemicals ${ }^{[17]}$. Nowadays, NMR gains more popularity in food applications due to its nondestructive nature ${ }^{[18]}$. NMR is very useful to study the physical and chemical properties of 
foods ${ }^{[19]}$. Despite the fact that NMR is less sensitive towards components present at a very low concentration, such as colorants and flavors, compared to other spectroscopic methods, it is still a very suitable method for food systems ${ }^{[19]}$. In food applications, NMR has been utilized in a wide range of studies, for example a study on the adsorption of caseinate in triacylglycerolin-water emulsions ${ }^{[20]}$, droplet size measurement in emulsions ${ }^{[21,22]}$, and electrostatic interaction between lysolecithin and chitosan in emulsions ${ }^{[23]}$. In the field of milk proteins, pfg-NMR has been used to study the diffusion of WPI and the gel structure of WPI ${ }^{[24,25]}$, the formation of protein and gum arabic coacervates ${ }^{[26]}$, the interaction of casein and maltodextrin ${ }^{[27]}$, the conjugation between corn fiber gum and milk proteins ${ }^{[28]}$, and the ligand binding to $\beta$ lactoglobulin [29].

Nevertheless, to our knowledge, there is limited research that utilizes NMR as a means to quantify protein-polysaccharide conjugates. By determining the diffusion coefficient of protein-polysaccharide mixtures, native protein, and native polysaccharides using NMR, it is possible to confirm the formation of protein-polysaccharide conjugates as well as to quantify the degree of interaction between proteins and polysaccharides. This is based on the fact that compounds with a different molecular weight have different diffusion behavior properties and these properties are altered upon interaction with other compounds. As interaction between proteins and polysaccharides results in new compounds with a higher molecular weight ${ }^{[30]}$, a change in the average diffusion coefficient as well as in the distribution width is expected.

In previous studies, chemical analyses using reagents such as Trinitrobenzenesulfonic acid (TNBS) ${ }^{[11,13]}$ and o-phthaldialdehyde (OPA) ${ }^{[10]}$ were performed to determine the degree of covalent interaction between proteins and polysaccharides by determining the residual amount of available amino groups in the conjugates. Whereas these methods are sensitive, they are also highly labor intensive and time consuming. NMR diffusometry, on the other hand, hardly requires any sample pretreatment and hence is less labor intensive.

In this study, pfg-NMR diffusometry was used to investigate the interaction of WPI and LMP as influenced by $\mathrm{pH}$, duration of the dry heat treatment (incubation time), and the concentration of LMP present in the conjugates. The diffusion coefficient and the distribution width of the WPI and LMP as influenced by these factors were reported. Moreover, the amount of free WPI and LMP-bound WPI in the conjugates could be determined. The outcome of this study was evaluated by comparing the results to those obtained through SDS-PAGE and TNBS analysis in a previous study [13]. 


\subsection{Materials}

The WPI was purchased from Davisco Foods International Inc. (Le Sueur, MN, USA). Protein analysis revealed that the WPI contained approximately $92.6 \%$ protein, whereby $85 \%$ of the protein is $\beta$-Lactoglobulin ${ }^{[31]}$. Low methoxyl pectin (LMP) (UnipectinOB700) was obtained from Cargill (Ghent, Belgium) and contained $89.6 \%$ of dry matter. Deuterium oxide $\left(\mathrm{D}_{2} \mathrm{O}>\right.$ 99.8\%) was purchased from Armar Chemicals (Switzerland).

\section{$2.2 \quad$ Methods}

\subsubsection{Conjugate preparation}

Conjugates were prepared from a 5\% (w/v) protein solution and 1\%(w/v) LM Pectin solution. A correction for the protein content and the dry matter was taken into account during the calculation of the WPI and LMP needed. The WPI and LMP were dissolved in distilled water and the $\mathrm{pH}$ of the solutions was adjusted to 7.0 with $1 \mathrm{~N} \mathrm{HCl}$ to avoid formation of ionic complexes that might form at lower $\mathrm{pH}$ during mixing. Both solutions were kept overnight in a refrigerator before mixing. The solutions were then mixed at four different ratios i.e. 1:0, 4:1, $2: 1$, and 1:1 (on weight basis) and frozen prior to freeze-drying.

The frozen samples were lyophilized (Alpha 1-2 LD plus, Christ) to remove all the water and obtain dry products. The freeze dried products were then incubated at a temperature of $60^{\circ} \mathrm{C}$ for 16 days in a desiccator containing saturated $\mathrm{NaCl}$ solution to keep the relative humidity at $74 \%$ ${ }^{[32]}$. During incubation, sampling was done at day $1,2,8$, and 16.

\subsubsection{High Resolution NMR}

High-resolution pulsed field gradient (pfg) NMR diffusion analysis was performed with a Bruker Avance III spectrometer operating at a ${ }^{1} \mathrm{H}$ frequency of $500.13 \mathrm{MHz}$ and equipped with a $5 \mathrm{~mm}$ DIFF30 gradient probe with a maximum gradient strength of $18 \mathrm{~T} / \mathrm{m}$. Pfg-NMR experiments were performed at $25^{\circ} \mathrm{C}$ using a (single) stimulated echo pulse sequence with bipolar gradient pairs. The samples $(565 \mu \mathrm{L})$ were filled in $5 \mathrm{~mm}$ diameter glass NMR tubes (Armar Chemicals, Switzerland) and were measured upon varying the gradient strength up to $12 \mathrm{~T} / \mathrm{m}$, while keeping the gradient duration $(\delta)$ and the diffusion delay $(\Delta)$ fixed at respectively $1.2 \mathrm{~ms}$ and $100 \mathrm{~ms}$. The samples were prepared by dissolving the powder in $10 \mathrm{mM} \mathrm{Na}$ - 
phosphate buffer ( $\mathrm{pH} 6.5$ and 7.2) and $5 \mathrm{mM}$ Na-acetate buffer ( $\mathrm{pH} 5.5$ and 5.0) in $\mathrm{D}_{2} \mathrm{O}$ to obtain a protein and pectin concentration of $20 \mathrm{mg} / \mathrm{mL}$ and $10 \mathrm{mg} / \mathrm{mL}$, respectively.

The obtained experimental echo intensity attenuation $(E)$ as a function of gradient strength $G$, which was typically sampled up to $95 \%$ decay, was then fitted to Eq. 1a using Matlab 7.5.0.342 (R2007b) software (Mathworks, Inc.). Hereby, a mass-weighted lognormal distribution $P_{v}$ of diffusion coefficients was assumed, whereas the echo decay was described by Eq. $1 \mathrm{~b}$ assuming free diffusion ${ }^{[33]}$. The corrected diffusion delay $\Delta^{\prime}$ in Eq.1b corresponds to $(\Delta-5 \delta / 16-\tau / 2)$, whereby $\tau$ stands for the delay time between the gradient pulses, which is equal to the sum of the gradient recovery delay and the $180^{\circ}$ pulse duration. The factor $s_{G}$ is a factor correcting for the non-rectangular shape of the applied pulsed field gradients (here equal to $2 / \pi$, corresponding to Sine bell shapes).

$$
\begin{aligned}
& E_{\text {expt }}=\int_{0}^{\infty} P_{v}\left(D_{i}\right) \cdot E\left(D_{i}\right) \cdot d D_{i} \\
& E\left(D_{i}\right)=\exp \left(-D_{i} \cdot \gamma^{2} \cdot\left(s_{G} \cdot G\right)^{2} \cdot \delta^{2} \cdot \Delta^{\prime}\right) \\
& P_{v}\left(D_{i}\right)=\frac{1}{\sqrt{2 \pi} \cdot D_{i} \cdot \ln \sigma_{g}} \cdot \exp \left(-\frac{\left(\ln \left(D_{i}\right)-\ln \left(D_{g}\right)\right)^{2}}{2 \cdot\left(\ln \sigma_{g}\right)^{2}}\right) \\
& D_{a}=D_{g} \cdot \exp \left(\frac{\left(\ln \sigma_{g}\right)^{2}}{2}\right) \\
& \sigma_{a}=\sqrt{D_{a}^{2} \cdot\left(\exp \left(\left(\ln \sigma_{g}\right)^{2}\right)-1\right)}
\end{aligned}
$$

A polydisperse population of diffusion coefficients with probability distribution $P_{v}$ was assumed because protein and pectin samples display a range of molar masses. For $P_{v}$, we assumed a lognormal mass-weighted diffusion coefficient distribution (Eq. 1c). The geometric mean diffusion coefficient $\left(D_{g}\right)$ and geometric standard deviation $\left(\sigma_{g}\right)$ of this distribution were converted to the arithmetic mean diffusion coefficient $\left(D_{a}\right)$ and arithmetic standard deviation $\left(\sigma_{a}\right)$ of the lognormal mass-weighted diffusion coefficient distribution using Eq. 1d and Eq. 1e. The degree of molecular interaction can be evaluated upon decomposing the WPI diffusion signal obtained from the WPI-LMP conjugate sample $E_{W P I, c o n j u g}$ into a free WPI fraction (whose decay corresponds to the experimentally obtained non-conjugated WPI signal $\left.E_{W P I, f r e e}\right)$ and a bound WPI fraction. The bound fraction can be determined upon 
estimating the diffusion signal of the reacted WPI $E_{W P I, b o u n d}$ using Eq. 2 and Matlab 7.5.0.342 (R2007b software, The Mathworks). As the molar mass of pectin is much larger as compared to WPI, the molar mass of the conjugate is mostly determined by pectin. Hence, the bound fraction can be determined assuming the experimentally obtained LMP diffusion signal to be a good approximation of the LMP-bound WPI diffusion signal as written in Eq. 3 using the Solver add-in (Microsoft Excel 2010). For WPI-LMP conjugates, the diffusion signal of dry heated WPI, which had the same incubation time as the WPI-LMP conjugates, was used as the diffusion signal of the free WPI. The WPI and LMP signal recorded at 0.6-1 ppm and 3.5-4.0 ppm were used in this calculation, respectively.

$$
\begin{aligned}
& E_{W P I, \text { conjug. }}=\varphi_{\text {free }} \cdot E_{W P I, \text { free }}+\varphi_{\text {bound }} \cdot E_{W P I, \text { bound }} \\
& E_{W P I, \text { conjug. }}=\left(1-\varphi_{\text {bound }}\right) \cdot E_{W P I, \text { free }}+\varphi_{\text {bound }} \cdot E_{L M P, \text { conjug }} .
\end{aligned}
$$

\subsubsection{Emulsion preparation}

$10 \%(\mathrm{w} / \mathrm{w})$ oil in water emulsions stabilized by either $0.5 \%(\mathrm{w} / \mathrm{w})$ WPI or $0.5 \%(\mathrm{w} / \mathrm{w})$ WPILMP mixtures (with or without dry heat treatment) were prepared. Emulsions were initially prepared by dissolving $0.5 \%$ of WPI or mixture of WPI-LMP. The aqueous phase was kept overnight in the fridge prior to the emulsion preparation to fully hydrate the hydrocolloid. Emulsions containing $10 \%(\mathrm{w} / \mathrm{w})$ of oil were prepared by adding $10 \mathrm{~g}$ of sunflower oil to $90 \mathrm{~g}$ of WPI solution. The mixture was then premixed using an IKA Ultra-turrax TV45 (Janke \& Kunkel, Staufen, Germany) at the highest speed (24000 rpm) for 1 minute. This was followed by homogenization using a Microfluidizer M110S for 2 minutes at 4 bar of compressed air pressure, corresponding to 560 bar of liquid pressure. The coil of the Microfluidizer was immersed in a water bath set at $55^{\circ} \mathrm{C}$.

\subsubsection{Electrophoretic mobility measurement}

The electrophoretic mobility of the emulsions stabilized by WPI and WPI-LMP mixtures was measured in $20 \mathrm{mM}$ of Na-acetate buffer at different $\mathrm{pH}$ values using a Zetasizer 2c (Malvern Ltd, UK). The emulsions were diluted 1000x in the buffers and let to stand overnight prior to the measurement. 


\section{Results and Discussions}

141

142

143

144

145

\subsection{Diffusion coefficient of native WPI and LMP}

In this study, a high gradient probe was needed due to the high molecular weight of WPI and LMP. From the NMR measurements, 1D spectra of native WPI and LMP were obtained (Fig. 1). By comparing the spectra of WPI and LMP, it was found that the signal observed at 0.6-1 ppm and 2.7-3.1 belonged to WPI, while the signal at 3.3-4.2 ppm and 5.2-5.5 ppm belonged to LMP. Thus, in a system containing both WPI and LMP, it was possible to distinguish the contribution of each biopolymer. The arithmetic mean diffusion coefficient $\left(D_{a}\right)$ and arithmetic standard deviation $\left(\sigma_{\mathrm{a}}\right)$ of WPI and LMP are reported in Table 1.

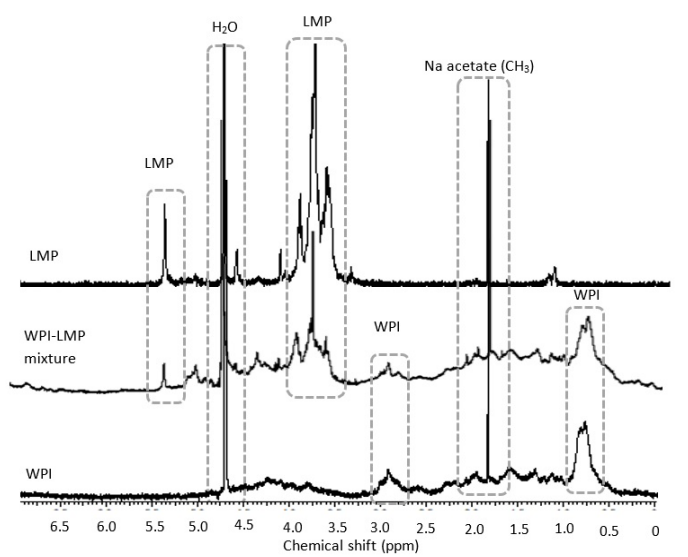

Figure 1. 1D ${ }^{1} \mathrm{H}$ spectrum of native WPI $(20 \mathrm{mg} / \mathrm{ml})$ and LMP $(10 \mathrm{mg} / \mathrm{ml})$ and WPI-LMP mixture (at 2:1 WPI:LMP ratio) in sodium phosphate buffer ( $\mathrm{pH} 7.2)$ containing $5 \mathrm{mM}$ sodium acetate, recorded at $25^{\circ} \mathrm{C}$.

The results presented in Table 1 and Figure 2 indicate that WPI $\left(D_{a}=7.13 \cdot 10^{-11} \mathrm{~m}^{2} / \mathrm{s}\right)$ diffuses much faster than LMP $\left(D_{a}=5.49 \cdot 10^{-11} \mathrm{~m}^{2} / \mathrm{s}\right)$. Furthermore, it was obvious that the arithmetic standard deviation, which represents the distribution width, of LMP was almost ten times bigger than the arithmetic standard deviation of WPI. The WPI sample used mainly consisted of $\beta$-lactoglobulin which has a molecular weight of $18 \mathrm{kDa}$ (monomer) with a narrow range of molecular weights. On the other hand, LMP has a bigger molecular weight of several hundred $\mathrm{kDa}{ }^{[34]}$ and a broader range of molecular weights. While the former explains the slow diffusion of LMP compared to WPI, the latter explains the broad diffusion coefficient distribution exhibited by LMP (Fig. 4). 
Table 1 Arithmetic mean diffusion coefficient $\left(D_{a}\right)$ and arithmetic standard deviation $\left(\sigma_{a}\right)$ value of WPI, LMP, and WPI-LMP conjugates, dry heat treated for 0 to 16 days obtained upon fitting Eq. 1 to the diffusion signal of the WPI and LMP contributions in the nonconjugated and conjugated samples.

\begin{tabular}{|c|c|c|c|c|c|c|c|}
\hline \multirow{2}{*}{$\begin{array}{l}\text { WPI:LMP } \\
\text { ratio } \\
(\mathrm{w} / \mathrm{w})\end{array}$} & \multirow{2}{*}{$\begin{array}{l}\text { Dry heat } \\
\text { treatment } \\
\text { time } \\
\text { (days) }\end{array}$} & \multirow[b]{2}{*}{$\mathrm{pH}$} & \multicolumn{2}{|c|}{ WPI (0.6-1.0 ppm) } & \multicolumn{2}{|c|}{ LMP (3.5-4.0 ppm) } & \multirow{2}{*}{$\begin{array}{l}\text { Free } \\
\text { WPI } \\
(\%)\end{array}$} \\
\hline & & & $\begin{array}{c}\mathrm{D}_{\mathrm{a}} \\
\left(\mathrm{x} 10^{-12} \mathrm{~m}^{2} / \mathrm{s}\right)\end{array}$ & $\begin{array}{c}\sigma_{\mathrm{a}} \\
\left(\mathrm{x} 10^{-12} \mathrm{~m}^{2} / \mathrm{s}\right)\end{array}$ & $\begin{array}{c}\mathrm{D}_{\mathrm{a}} \\
\left(\mathrm{x} 10^{-12} \mathrm{~m}^{2} / \mathrm{s}\right)\end{array}$ & $\begin{array}{c}\sigma_{\mathrm{a}} \\
\left(\mathrm{x} 10^{-12} \mathrm{~m}^{2} / \mathrm{s}\right)\end{array}$ & \\
\hline $1: 0$ & 0 & 7.2 & 71.3 & 14.2 & & & \\
\hline $0: 1$ & 0 & 7.2 & & & 54.9 & 161.0 & \\
\hline \multirow{5}{*}{$2: 1$} & 0 & 7.2 & 70.5 & 35.2 & 54.5 & 59.2 & 87 \\
\hline & 1 & 7.2 & 65.1 & 31.4 & 58.0 & 75.3 & 73 \\
\hline & 2 & 7.2 & 64.2 & 35.4 & 49.4 & 57.3 & 66 \\
\hline & 8 & 7.2 & 62.7 & 41.4 & 47.0 & 57.2 & 64 \\
\hline & 16 & 7.2 & 59.7 & 35.4 & 47.5 & 52.8 & 63 \\
\hline \multirow{2}{*}{$2: 1$} & 0 & 5.5 & 59.5 & 33.7 & 51.9 & 81.2 & 75 \\
\hline & 0 & 5.0 & 56.7 & 37.0 & 65.5 & 137.0 & 52 \\
\hline $4: 1$ & 8 & 7.2 & 71.5 & 36.3 & 64.1 & 59.6 & 61 \\
\hline $1: 1$ & 16 & $6.5-6.8$ & 88.0 & 141.0 & 54.0 & 150.0 & 56 \\
\hline
\end{tabular}
value of WPI, which has been dry heat-treated for 0-16 days obtained upon fitting Eq. 1 to the diffusion signal of the WPI contributions in the NMR spectrum (at 0.6-1 ppm and 2.7-3.1 ppm, resp.) of the samples.

\begin{tabular}{|c|c|c|c|c|}
\hline \multirow{2}{*}{$\begin{array}{c}\text { Dry heat } \\
\text { treatment } \\
\text { time (days) }\end{array}$} & \multicolumn{2}{|c|}{$0.6-10 \mathrm{ppm}$} & \multicolumn{2}{c|}{$2.7-3.1 \mathrm{ppm}$} \\
\cline { 2 - 5 } & $\begin{array}{c}\mathrm{D}_{\mathrm{a}} \\
\left(\mathrm{x} 10^{-12} \mathrm{~m}^{2} / \mathrm{s}\right)\end{array}$ & $\begin{array}{c}\sigma_{\mathrm{a}} \\
\left(\times 10^{-12} \mathrm{~m}^{2} / \mathrm{s}\right)\end{array}$ & $\begin{array}{c}\mathrm{D}_{\mathrm{a}} \\
\left(\times 10^{-12} \mathrm{~m}^{2} / \mathrm{s}\right)\end{array}$ & $\begin{array}{c}\sigma_{\mathrm{a}} \\
\left(\times 10^{-12} \mathrm{~m}^{2} / \mathrm{s}\right)\end{array}$ \\
\hline 0 & 71.3 & 14.2 & 68.5 & 5.0 \\
\hline 1 & 71.1 & 13.6 & 74.8 & 20.7 \\
\hline 2 & 71.9 & 12.8 & 73.9 & 21.0 \\
\hline 8 & 73.9 & 25.6 & 80.5 & 35.3 \\
\hline
\end{tabular}




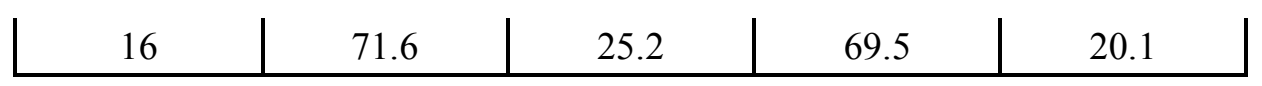

176 In order to evaluate the influence of dry heat treatment on WPI, freeze dried WPI was dry heat 177 treated up to 16 days. Previously, SDS-PAGE analysis revealed that a limited protein 178 polymerization occurred in dry heat treated WPI ${ }^{[13]}$. Polymerization of protein leads to the 179 formation of molecules with higher molecular weight ${ }^{[35]}$ that diffuse slower. Table 2 revealed 180 that upon dry heat treatment of WPI, the arithmetic mean diffusion coefficient of WPI did not decrease significantly. On the other hand, it can be seen in Table 2 that the distribution width of WPI became two times broader after 8 days of dry heat treatment, which might be due to polymerization of some of the protein molecules in WPI.

Hereinafter, the discussion of the results obtained on dry heat-treated mixtures of WPI and LMP is divided into 3 parts, which describe the influence of $\mathrm{pH}$, incubation time, and WPI to LMP ratio.

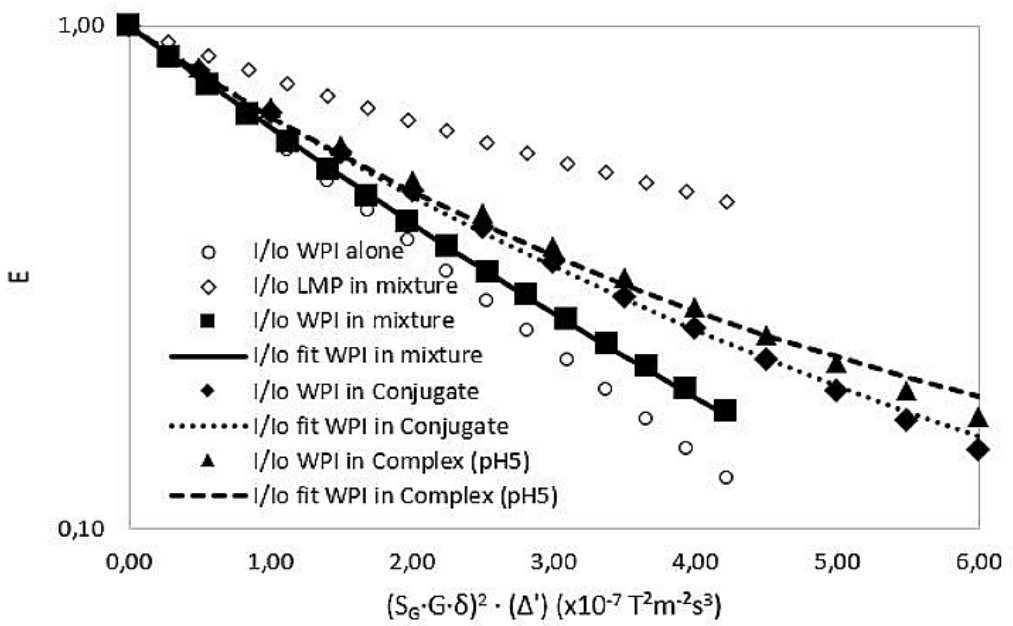

Figure 2. Decomposition of the WPI diffusion signal in the presence of LMP into the calculated protein diffusion signal without pectin (lyophilized/dry heat treated WPI only $(1: 0) ; 0$ ) and the calculated pectin signal ( $\diamond)$ in WPI-LMP conjugates of ratio 2:1 after 0 (mixture) and 16 days (conjugates) of dry heat treatment (measured at $\mathrm{pH} 7.2$ ), as well as in a 


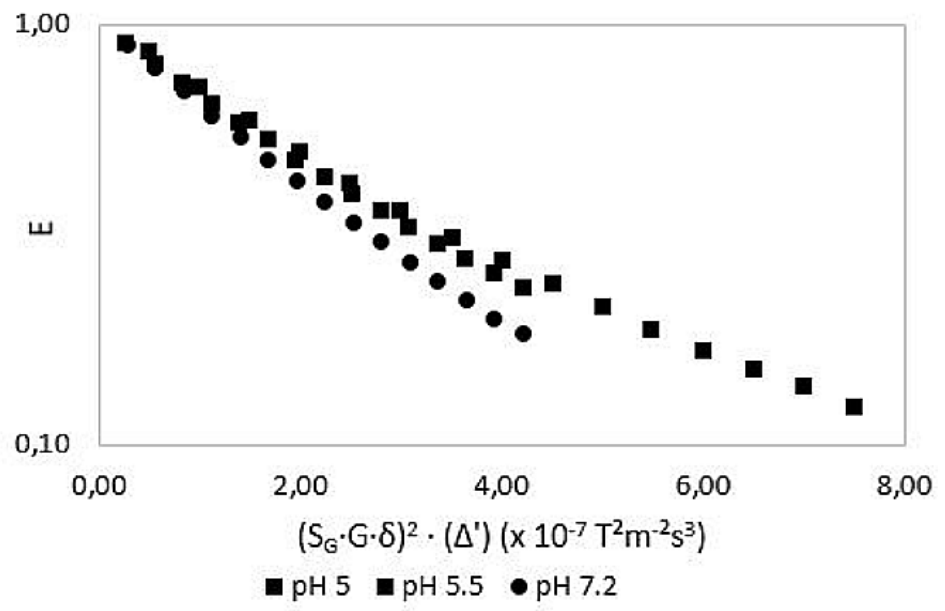

195

Figure 3. Diffusion echo decay of the WPI signal (0.6-1.0 ppm) in WPI-LMP mixtures (without dry heat treatment, ratio 2:1, Day 0) as a function of $\mathrm{pH}$.

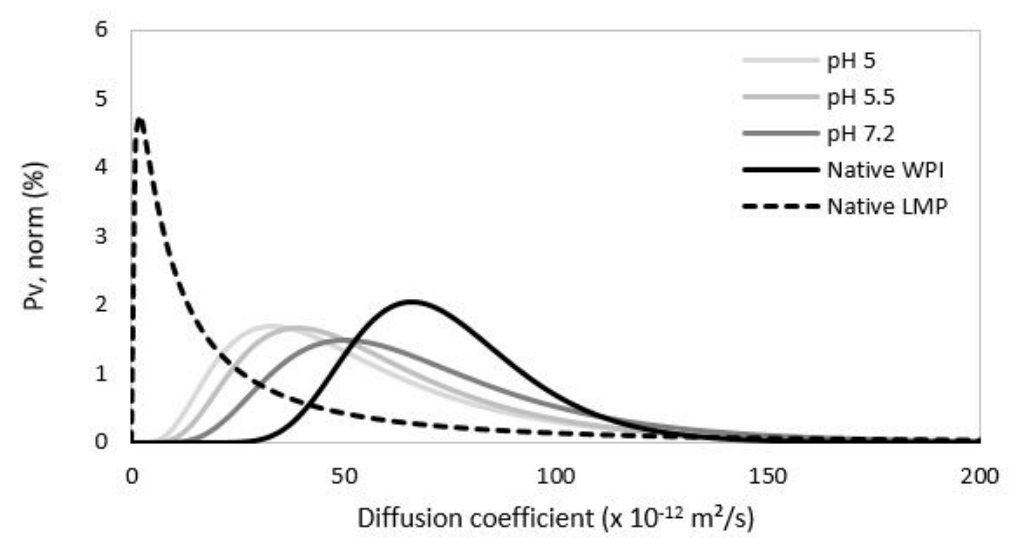

Figure 4. The normalized lognormal mass-weighted probability density function of the diffusion coefficient of the native WPI (0.6-1.0 ppm) and LMP (3.5-4.0 ppm) as well as of the WPI and LMP in a 2:1 (w/w) mixture of WPI-LMP prepared at $\mathrm{pH} 7.2,5.5$, and 5.0.

In this section, the influence of $\mathrm{pH}$ on the diffusion coefficient of WPI and LMP in a mixture containing both biopolymers is discussed. For this purpose, mixtures of WPI and LMP with ratio of 2:1 were prepared at a pH close to the IEP of WPI (5.0 and 5.5) and at relatively neutral $\mathrm{pH}$ (7.2). It is indeed well known that the electrostatic interaction between WPI and LMP is governed by $\mathrm{pH}$. A typical 1D ${ }^{1} \mathrm{H}-\mathrm{NMR}$ spectrum of a system containing both WPI and LMP can be seen in Fig. 1. Fig.3 shows that when the $\mathrm{pH}$ was decreased to around the IEP of WPI, the diffusional attenuation of $b$ WPI in the mixture decreased less rapidly which indicated 
slower diffusion due to molecular interaction between WPI and LMP. At $\mathrm{pH}$ 5.0, this effect was more pronounced than at $\mathrm{pH}$ 5.5, which indicates that there was more WPI bound to LMP at $\mathrm{pH} 5.0$ than at $\mathrm{pH}$ 5.5. This was supported by the value of the arithmetic mean diffusion coefficient of WPI in the mixtures (2:1 Day 0) prepared at $\mathrm{pH} 7.2,5.5$ and 5.0, which was $7.05 \cdot 10^{-11} \mathrm{~m}^{2} / \mathrm{s}, 5.95 \cdot 10^{-11} \mathrm{~m}^{2} / \mathrm{s}$, and $5.67 \cdot 10^{-11} \mathrm{~m}^{2} / \mathrm{s}$, respectively (Table 1 ). These values were smaller compared to that of native WPI $\left(7.13 \cdot 10^{-11} \mathrm{~m}^{2} / \mathrm{s}\right)$. Hence, for the WPI in the presence of LMP, the arithmetic mean diffusion coefficient decreased as the $\mathrm{pH}$ was lowered towards the protein's IEP. The distribution width of WPI increased from $1.42 \cdot 10^{-11} \mathrm{~m}^{2} / \mathrm{s}$ (native WPI) to about $3.5 \cdot 10^{-11} \mathrm{~m}^{2} / \mathrm{s}$ in the presence of LMP. On the other hand, the diffusion coefficient of the LMP (3.5-4.0 ppm) at pH 7.2, 5.5, and 5.0 was $5.45 \cdot 10^{-11} \mathrm{~m}^{2} / \mathrm{s}, 5.19 \cdot 10^{-11} \mathrm{~m}^{2} / \mathrm{s}$, and $6.55 \cdot 10^{-}$ ${ }^{11} \mathrm{~m}^{2} / \mathrm{s}$, respectively. Strong electrostatic interaction between biopolymers reduces their diffusion coefficient ${ }^{[26]}$. Hence, the diffusion coefficient of LMP at pH 5.0 was expected to be the smallest. However, it was found that at $\mathrm{pH} 5.0$ the arithmetic mean diffusion coefficient of LMP was higher than that at pH 7.2 and 5.5. However, it has to be noted that the LMP peak at $\mathrm{pH} 5.0$ exhibited a broader diffusion coefficient distribution $\left(\sigma_{\mathrm{a}}=1.37 \cdot 10^{-10} \mathrm{~m}^{2} / \mathrm{s}\right)$ than that at pH $7.2\left(\sigma_{\mathrm{a}}=5.92 \cdot 10^{-11} \mathrm{~m}^{2} / \mathrm{s}\right)$ and $5.5\left(\sigma_{\mathrm{a}}=8.12 \cdot 10^{-11} \mathrm{~m}^{2} / \mathrm{s}\right)($ Table 1$)$.

From the decomposition of the WPI signal in the mixture into the calculated WPI diffusion signal without LMP (lyophilized/dry heat treated WPI only) and the calculated pectin signal with LMP (WPI-LMP conjugates/ WPI-LMP complex) (Figure 2), the fraction of unreacted WPI or free WPI in the mixture was calculated. At $\mathrm{pH} 7.2$, there was approximately $13 \%$ of WPI bound to LMP and 87\% of free WPI. At pH 5.5 and 5.0, the amount of LMP-bound WPI increased, as expected, to $39 \%$ at $\mathrm{pH} 5.5$ and $44 \%$ as the $\mathrm{pH}$ was further decreased to 5.0 . This result explains the trend observed in the diffusion coefficient of WPI previously mentioned. In addition, it can be noticed in Figure 2 that the diffusion signal of WPI shifted further towards the diffusion signal of LMP as more WPI was bound to LMP. 


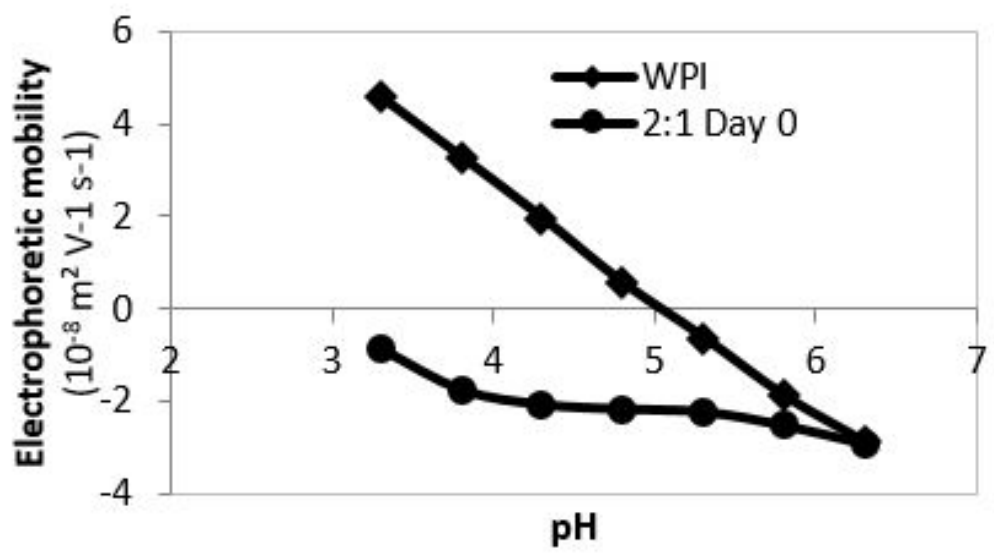

Figure 5. Electrophoretic mobility profile of emulsions stabilized by native WPI ( $\bullet$ ) and by a 2:1 (w/w) WPI-LMP mixture (without dry heat treatment) $(\bullet)$.

237 These results were further supported by electrophoretic mobility measurement of emulsions 238 stabilized by either WPI only or a WPI-LMP mixture at different $\mathrm{pH}$ values. Figure 5 indicates 239 that at $\mathrm{pH} 5.0-5.5$, the EM of emulsions stabilized by a WPI-LMP mixture was more negative 240 than that stabilized by WPI only. This was due to the presence of the negatively charged LMP 241 at the surface of the oil droplets via electrostatic interaction with the positive patches on WPI. 242 At a higher $\mathrm{pH}$, electrostatic attraction between WPI and LMP is not favorable due to charge 243 repulsion since both biopolymers carry a net negative charge ${ }^{[7]}$. . Hence, it might be expected 244 that there would be no WPI bound to LMP at pH 7.2. However, the NMR results clearly indicated some interaction between WPI and LMP, even at pH 7.2. This is thought to be due to a weak electrostatic attraction between the anionic pectin and positively charged regions of 247 the WPI ${ }^{[36,37]}$. The same authors also mention that hydrogen bonds are also involved in the interaction between WPI and pectin at a pH slightly above the IEP of the protein, albeit to a lesser extent. 


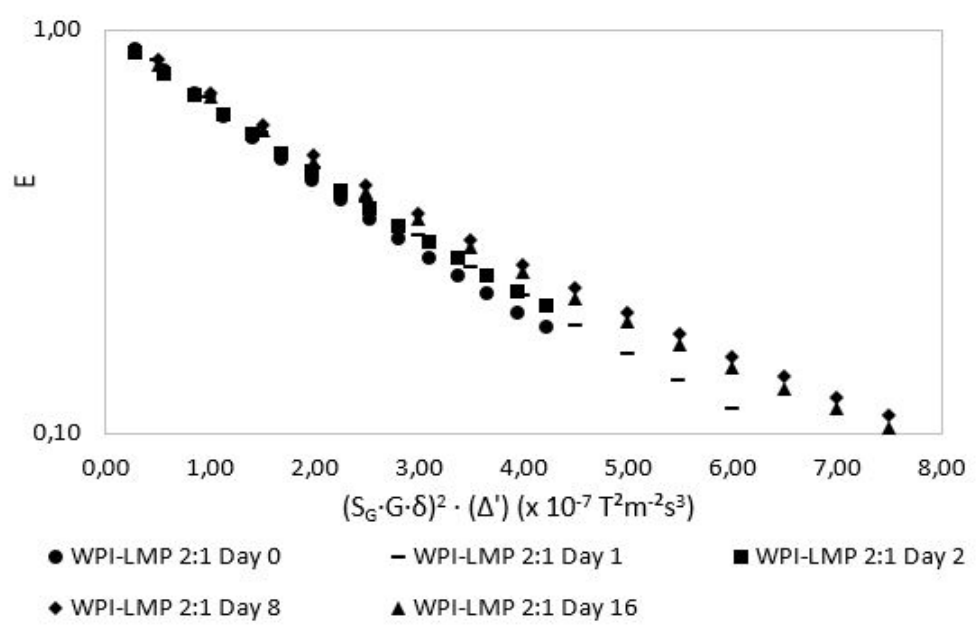

251

Figure 6. Diffusion echo decay of the WPI signal (0.6-1.0 ppm) in WPI-LMP conjugates prepared at $\mathrm{pH} 7.2$ as a function of dry heat incubation time.

254

255

The interaction between WPI and LMP as influenced by incubation time was determined at $\mathrm{pH}$ 7.2 to minimize the electrostatic interaction which might cause an overestimation of the results. Figure 6 shows the diffusion echo decay of the LMP and WPI signal in the conjugates as a function of dry heat incubation time. As it can be observed, the longer the dry heat incubation time, the more the graphs shifted upward. However, it can be seen that the diffusion echo decay of the WPI in WPI-LMP conjugates obtained after 8 or 16 days of dry heat treatment almost coincided. The observed upward shift was due to the increase of the molar weight of WPI/LMP caused by the conjugation. Upon dry heat treatment, amino groups of WPI become covalently linked to the carbonyl groups of LMP through a Maillard type reaction ${ }^{[38]}$. A longer incubation time is expected to result in a higher degree of interaction between WPI and LMP, leading to the reduction of the free WPI fraction in the system.

Table 1 reveals that dry heat treatment for one day decreased the arithmetic mean diffusion coefficient of WPI from $7.05 \cdot 10^{-11} \mathrm{~m}^{2} / \mathrm{s}$ (Day 0 ) to $6.51 \cdot 10^{-11} \mathrm{~m}^{2} / \mathrm{s}$. Further incubation of the conjugates resulted in a further reduction of the WPI diffusion coefficient to $6.42 \cdot 10^{-11} \mathrm{~m}^{2} / \mathrm{s}$ after 2 days of dry heat treatment, $6.27 \cdot 10^{-11} \mathrm{~m}^{2} / \mathrm{s}$ after 8 days of dry heat treatment, and $5.97 \cdot 10^{-}$ ${ }^{11} \mathrm{~m}^{2} / \mathrm{s}$ after 16 days of dry heat treatment. A change in the distribution width was also observed (Table 1). Compared to native WPI, the WPI signal in conjugates had a broader distribution width (Fig.7). As for LMP, the trend was similar: the diffusion coefficient of LMP decreased as the incubation time was prolonged. The LMP signal had a diffusion coefficient of $5.45 \cdot 10^{-}$ 

treatment for $0,1,2,8$ and 16 days, respectively.

275

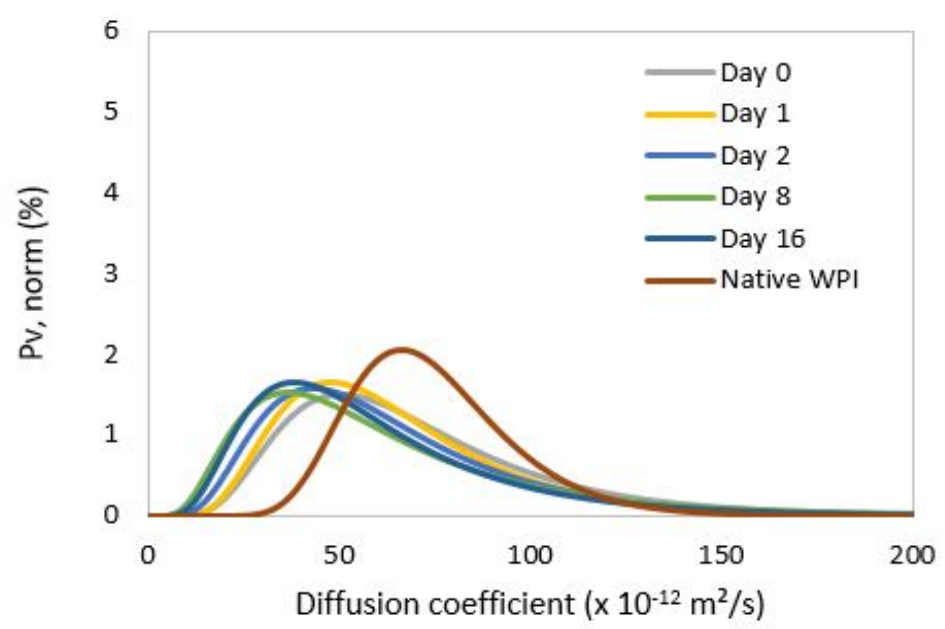

Figure 7. The normalized lognormal mass-weighted probability density function of the diffusion coefficient of WPI (0.6-1.0 ppm) in WPI-LMP conjugates which were incubated for $0,1,2,8$, and 16 days.

Signal decomposition of the WPI signal in the conjugates obtained after 16 days of dry heat treatment into the WPI signal without and with LMP can be seen in Fig. 3. From the calculation, it was found that in the unheated WPI-LMP mixture (Day 0), there was already 13\% of WPI bound to LMP which was most probably formed due to weak electrostatic interaction between WPI and LMP. It was observed that upon dry heat treatment, the percentage of free WPI decreased from $87 \%$ (Day 0 ) to $73 \%, 66 \%, 64 \%$, and $63 \%$ after $1,2,8$, and 16 days of dry heat treatment. Table 1 reveals that the WPI-LMP mixtures were characterized by rapid formation of conjugates in the first 2 days of dry heat treatment. Extension of the dry heat treatment for more than 2 days did not considerably increase the rate of conjugation.

\subsection{Influence of WPI to LMP ratio}

The influence of the WPI to LMP ratio was also investigated. In this study, the concentration of the WPI was kept constant at all ratios, while the concentration of the LMP was varied. WPILMP conjugates with four different WPI to LMP ratios, namely 1:0, 4:1, 2:1, and 1:1 were prepared. The conjugates with ratio of 1:0, 4:1, and 2:1 were incubated for 8 days, while conjugates with ratio 1:1 were incubated for 16 days. This comparison was made since an extension of the dry heat treatment time from 8 days to 16 days did not considerably increase 
302

303

the amount of conjugates. The conjugates were measured at $\mathrm{pH} 7.2$ in a phosphate buffer containing $5 \mathrm{mM}$ of sodium acetate, except for the conjugates with ratio 1:1. The latter was measured in $\mathrm{D}_{2} 0$ containing $5 \mathrm{mM}$ sodium acetate with a final $\mathrm{pH}$ of approximately $6.5-6.8$.

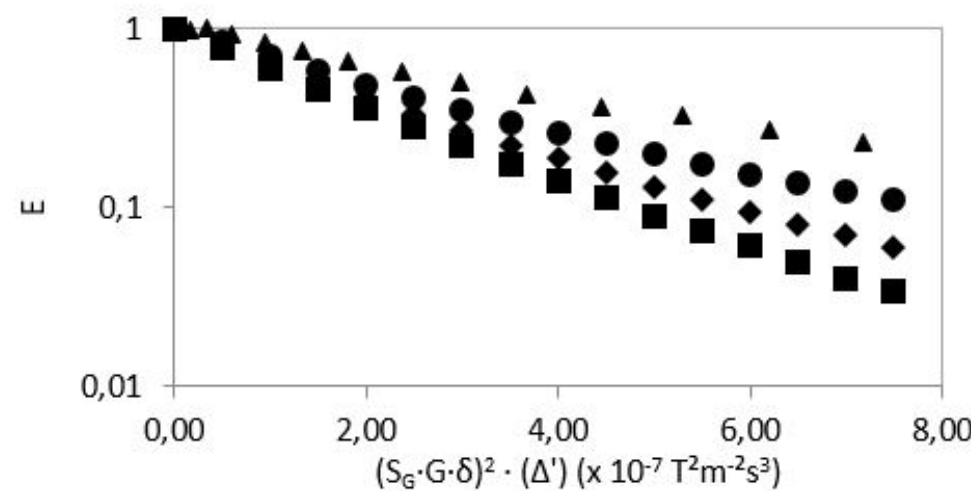

-WPI-LMP 1:0 • WPI-LMP 4:1 • WPI-LMP 2:1 ^ WPI-LMP 1:1

Figure 8. Diffusion echo decay of the WPI signal (0.6-1.0 ppm) in WPI-LMP conjugates at ratio $1: 0,4: 1,2: 1$, and 1:1 prepared at $\mathrm{pH} 7.2$ after 8 days (ratio 1:0, 4:1, and 2:1) or 16 days (ratio 1:1) of dry heat incubation time.

The diffusion echo decay of the LMP and WPI signal as influenced by the WPI to LMP ratio can be seen in Figure 8. In the case of LMP, the graphs shifted upward as the concentration of LMP was higher. The same trend was observed for WPI. It can be seen in Table 1 that the conjugates with ratio $1: 0,4: 1,2: 1$, and $1: 1$ had an arithmetic mean diffusion coefficient of 7.45 , $7.15,6.27$, and $8.80 \cdot 10^{-11} \mathrm{~m}^{2} / \mathrm{s}$, respectively, when considering the WPI peak at 0.6-1.0 ppm. On the other hand, the arithmetic mean standard deviation of the distribution of the conjugates was reported to be $2.56,3.63,4.14$, and $14.1 \cdot 10^{-11} \mathrm{~m}^{2} / \mathrm{s}$ for the conjugates with ratio of $1: 0,4: 1$, $2: 1$, and $1: 1$, respectively. 


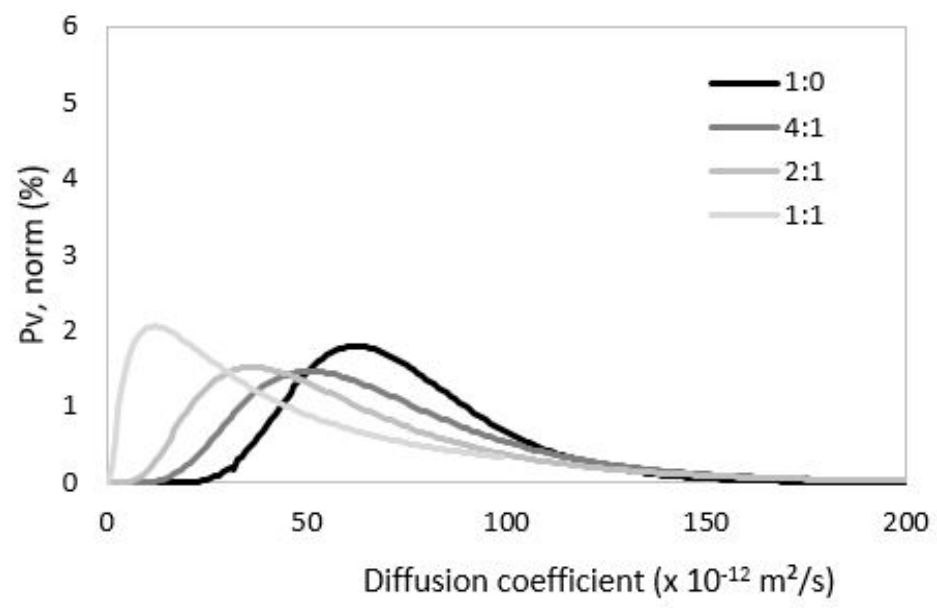

Figure 9. The normalized lognormal mass-weighted probability density function of the diffusion coefficient of the WPI (0.6-1.0 ppm) signal in WPI-LMP conjugates with ratio of $1: 0,4: 1,2: 1$, and $1: 1$.

While it was expected that the diffusion coefficient of the WPI signal at ratio 1:1 would be the smallest, it was found that it had the biggest diffusion coefficient. Nevertheless, it has to be noted that the WPI signal at this ratio exhibited a much broader distribution width than the others (Fig. 9). A similar behavior was previously observed in a mixture of WPI and LMP prepared at pH 5 (paragraph 3.2). Besides, Figure 9 clearly indicates that the distribution mode (i.e., the diffusion coefficient corresponding to the maximum in the distribution curve) gradually decreased as the LMP concentration increased.

The signal decomposition and calculation of the free WPI fraction in the conjugates confirmed the phenomena explained above (Table 1). After 8 days of dry heat treatment, there was $75 \%$ of unreacted WPI in the conjugates with ratio 4:1. By Increasing the proportion of LMP in the conjugates to a WPI:LMP ratio of $2: 1$, the fraction of free WPI present in the conjugate decreased to $64 \%$. Finally, at a ratio 1:1 almost half of the WPI was bound to LMP (Table 1). Therefore, it can be concluded that increasing the concentration of LMP in the WPI-LMP conjugates resulted in a higher degree of conjugation between WPI and LMP.

\subsection{Discussion}

329 The current study was performed to investigate the capabilities of diffusion NMR as a tool to confirm and to quantify the formation of WPI-LMP conjugates or complexes, as an alternative of chemical analysis. Based on the aforementioned results, it can be seen that interaction of WPI and LMP, through both electrostatic and covalent interaction, resulted in the decrease of 
the arithmetic mean diffusion coefficient and the increase of the arithmetic mean standard deviation of WPI. The latter is due to the broad molecular weight range of LMP. Since native WPI has a very narrow distribution width due to its uniform molecular weight, its interaction with LMP dramatically influenced the average diffusion coefficient and distribution width of WPI. On the other hand, the diffusion coefficient properties of the LMP were much less affected by the presence of bound WPI. The results also indicate that as whey protein interacted with LMP, it acquired the diffusion properties of LMP, which points to the fact that smaller WPI molecules become bound to larger LMP molecules according to a pearls on a necklace geometry. For the sake of completeness, it has to be mentioned that an increase of the diffusion coefficient was sometimes observed when there was strong interaction between the WPI and LMP, as was the case in WPI-LMP conjugates with a ratio of $1: 1$ which was dry heat treated for 16 days. This phenomenon was due to the fact that the increased distribution width sometimes overruled the expected effect on the arithmetic mean value: in fact, considering a lognormal distribution, the arithmetic mean increases with increasing distribution width for a constant geometric mean.

In the case of protein-polysaccharides conjugates or complexes, the degree of interaction is an essential parameter as it is highly related to the functionality of these conjugates or complexes [39]. Furthermore, research on improving the functionality of proteins through interaction with other biopolymers is currently receiving a lot of attention ${ }^{[40]}$. It has been reported that the yield of the conjugation (dry heat treatment) (i.e. the degree of interaction) has an influence on the emulsifying activity and heat stability of protein-pectin conjugates ${ }^{[40-42]}$. The better emuslsifying acitivity of the protein upon dry heat treatment is attributed to the present of polysaccharides. Upon emulsification the proteins are absorbed to the surface of oil droplets and thus polysaccharides are carried by the proteins and become available on the surface of the oil droplets ${ }^{[12]}$. Adsorption of polysaccharides to the surface of oil droplet is not possible or very limited unless it is bound to proteins. The present of polysaccharides on the surface of oil droplet stabilizes the oil droplets in an oil in water emulsion system by providing additional viscoelastic layer on the surface of oil droplet and through steric hindrance ${ }^{[12,43]}$. Hereby, knowing the degree of interaction or the minimum amount of polysaccharides required to be linked to proteins and present on the surface of oil droplet is essential to have a good understanding on the mechanism of protein-polysacharides functionality. Since the degree of interaction is influenced by some factors such as the biopolymer concentration, $\mathrm{pH}$, time, relative humidity and water activity (for dry heat treatment), and temperature, quantifying the 
degree of interaction will help finding the optimum conditions for the preparation of conjugates or complexes with the desired functional properties. In addition, it will be also possible to determine the minimum degree of interaction required to achieve optimum functional properties.

The results obtained from NMR measurements were generally in agreement with those obtained by chemical analysis. SDS-PAGE analysis performed in a previous study showed that dry heat treatment of WPI-LMP mixtures resulted in the formation of high molecular weight compounds (WPI-LMP conjugates) [13]. In the same study, the degree of interaction between WPI and LMP was determined by measuring the availability of the primary amino group of WPI in the conjugates as a function of incubation time using the TNBS method ${ }^{[13]}$. Compared to the results obtained from TNBS measurement ${ }^{[13]}$, the degree of interaction between WPI and LMP obtained using NMR was higher: TNBS analysis indicated that after 16 days of dry heat treatment, WPI-LMP conjugates with a ratio 2:1 had a degree of conjugation of approximately $15 \%$. However, it has to be noted that $\beta$-lactoglobulin has 15 lysine residues. Thus, if only one out of the 15 amino groups reacts (i.e. $6.67 \%$ based on the number of free amino groups), all WPI may become bound to LMP (i.e. 100\% based on diffusion NMR). Despite of showing a different magnitude of the degree of interaction, the general trend obtained from the NMR measurements was comparable with that obtained from the TNBS measurements in which the rate of WPI and LMP interaction was intensive in the first 4 days and decreased upon extension of the dry heat treatment incubation time. Furthermore, the TNBS analysis results also showed that the degree of interaction increased as the concentration of LMP in the mixture was higher, which was also in line with the results of NMR measurements.

A lot of useful information can be extracted from NMR measurements, such as the molecular structure, diffusion coefficient, and distribution width, which are related to the molecular weight distribution of the targeted compound ${ }^{[26-29,39,44]}$. Some studies related to milk protein employed NMR to confirm the interaction between the protein and other compounds using only the NMR spectra ${ }^{[26,27]}$ or by determining the diffusion coefficient of the protein ${ }^{[28,29]}$. In fact, the results obtained from NMR were useful to quantify the degree of interaction between proteins and polysaccharides or other biopolymers. This was performed by decomposing the protein signal of the conjugates or complexes into the WPI signal without pectin (native state) and the pectin signal of the mixture using Eq. 2 and 3. 
The results of this study indicate that NMR diffusometry is a promising method to study and quantify protein and polysaccharide interaction, in which the results are comparable to that obtained from chemical analysis. Whereas chemical analysis, e.g. using the TNBS and OPA method, cannot be used for protein-polysacccharide electrostatic interaction, NMR measurement works well for both electrostatic and covalent interactions. Compared to chemical analysis, e.g. using the TNBS method, which is time consuming and labor intensive, this method is more user-friendly and the sample preparation time is relatively short. Furthermore, the required amount of sample for the NMR analysis is low and no sample pretreatment is needed.

\section{Conclusions}

Whereas the diffusion properties of LMP were less affected, the results showed that interaction between WPI and LMP, either through electrostatic or covalent interaction, decreased the diffusional speed of WPI. Deconvolution of the experimentally acquired signal of complexes or conjugates enabled the quantification of the fraction of free and reacted WPI in mixtures and conjugates of WPI-LMP. The results obtained from the NMR analysis were also found to be comparable to those obtained by alternative methods, such as SDS-page, spectrophotometric determination of the concentration of available free amino groups by the TNBS method, as well as electrophoretic mobility evaluation, as reported by Setiowati et al. ${ }^{[13]}$. However, NMR measurements are relatively user friendly and require relatively little sample preparation. Some work dealing with the application of NMR to study the interaction of protein with other compounds has been published ${ }^{[26-28,39]}$. However, these studies did not perform any signal decomposition to quantify the degree of interaction, which is an important factor for the application of conjugates or complexes. Future application of this system to determine the molecular weight of the complexes or conjugates would be very interesting.

\section{Acknowledgement}

This research has been supported by the Indonesian Endowment Fund for Education (LPDP Indonesia). The $500 \mathrm{MHz}$ NMR equipment was funded by the Hercules Foundation (contract number: AUGE09/006). Davy Sinnaeve thanks the Research Foundation - Flanders for a postdoctoral fellowship. 
[1] T. Harnsilawat, R. Pongsawatmanit, D.J. McClements, Stabilization of model beverage cloud emulsions using protein- polysaccharide electrostatic complexes formed at the oil-water interface, Journal of agricultural and food chemistry 54(15) (2006) 5540-5547.

431 [2] M. Akhtar, E. Dickinson, Whey protein-maltodextrin conjugates as emulsifying agents: an 432 alternative to gum arabic, Food Hydrocolloids 21(4) (2007) 607-616.

433 [3] C.M. Bryant, D.J. McClements, Molecular basis of protein functionality with special consideration 434 of cold-set gels derived from heat-denatured whey, Trends in Food Science \& Technology 9(4) (1998) 435 143-151.

436 [4] A.F. Esfanjani, S.M. Jafari, E. Assadpour, Preparation of a multiple emulsion based on pectin-whey protein complex for encapsulation of saffron extract nanodroplets, Food chemistry 221 (2017) 19621969.

439 [5] Z. Teng, Y. Luo, Q. Wang, Carboxymethyl chitosan-soy protein complex nanoparticles for the

[6] A. Ye, Complexation between milk proteins and polysaccharides via electrostatic interaction: principles and applications-a review, International Journal of Food Science \& Technology 43(3) (2008) 406-415.

[7] Y.S. Gu, E.A. Decker, D.J. McClements, Influence of $\mathrm{pH}$ and carrageenan type on properties of $\beta$ lactoglobulin stabilized oil-in-water emulsions, Food Hydrocolloids 19(1) (2005) 83-91.

[8] A. Benichou, A. Aserin, R. Lutz, N. Garti, Formation and characterization of amphiphilic conjugates of whey protein isolate (WPI)/xanthan to improve surface activity, Food Hydrocolloids 21(3) (2007) 379-391.

[9] A. Benichou, A. Aserin, N. Garti, W/O/W double emulsions stabilized with WPI-polysaccharide complexes, Colloids and Surfaces A: Physicochemical and Engineering Aspects 294(1) (2007) 20-32.

[10] T.J. Wooster, M.A. Augustin, $\beta$-Lactoglobulin-dextran Maillard conjugates: Their effect on interfacial thickness and emulsion stability, Journal of Colloid and Interface Science 303(2) (2006) 564572.

[11] L. Jimenez-Castano, M. Villamiel, R. López-Fandiño, Glycosylation of individual whey proteins by Maillard reaction using dextran of different molecular mass, Food Hydrocolloids 21(3) (2007) 433-443.

[12] A. Kato, Industrial applications of Maillard-type protein-polysaccharide conjugates, Food Science and Technology Research 8(3) (2002) 193-199.

[13] A.D. Setiowati, L. Vermeir, J. Martins, B. De Meulenaer, P. Van der Meeren, Improved heat stability of protein solutions and $\mathrm{O} / \mathrm{W}$ emulsions upon dry heat treatment of whey protein isolate in the presence of low-methoxyl pectin, Colloids and Surfaces A: Physicochemical and Engineering Aspects 510 (2016) 93-103.

[14] L. Jiménez-Castaño, R. López-Fandiño, A. Olano, M. Villamiel, Study on $\beta$-lactoglobulin glycosylation with dextran: effect on solubility and heat stability, Food Chemistry 93(4) (2005) 689695.

[15] N. Neirynck, P. Van der Meeren, S. Bayarri Gorbe, S. Dierckx, K. Dewettinck, Improved emulsion stabilizing properties of whey protein isolate by conjugation with pectins, Food Hydrocolloids 18(6) (2004) 949-957.

[16] H. Salminen, J. Weiss, Electrostatic adsorption and stability of whey protein-pectin complexes on emulsion interfaces, Food Hydrocolloids 35 (2014) 410-419.

[17] P.Y. Ghi, D.J. Hill, A.K. Whittaker, PFG-NMR measurements of the self-diffusion coefficients of water in equilibrium poly (HEMA-co-THFMA) hydrogels, Biomacromolecules 3(3) (2002) 554-559.

[18] M. Voda, J. Van Duynhoven, Characterization of food emulsions by PFG NMR, Trends in food science \& technology 20(11) (2009) 533-543.

[19] S. Ablett, Overview of NMR applications in food science, Trends in Food Science \& Technology 3 (1992) 246-250.

[20] Y. Mine, Phosphorus-31 nuclear magnetic resonance study on adsorption behaviour of caseinate in triacylglycerol-in-water emulsions, Journal of agricultural and food chemistry 45(1) (1997) 68-73. 

Effect of molecular exchange on water droplet size analysis in W/O emulsions as determined by diffusion NMR, Journal of Colloid and Interface Science 463 (2016) 128-136. [22] A. Métais, F. Mariette, Determination of water self-diffusion coefficient in complex food products weighted spin-echo sequence, Journal of Magnetic Resonance 165(2) (2003) 265-275.

[23] Y. Kwamman, B. Mahisanunt, S. Matsukawa, U. Klinkesorn, Evaluation of electrostatic interaction between lysolecithin and chitosan in two-layer tuna oil emulsions by Nuclear Magnetic Resonance (NMR) spectroscopy, Food biophysics 11(2) (2016) 165.

[24] P. Croguennoc, T. Nicolai, M.E. Kuil, J.G. Hollander, Self-diffusion of native proteins and dextran in heat-set globular protein gels, The Journal of Physical Chemistry B 105(24) (2001) 5782-5788.

[25] C. Le Bon, T. Nicolai, M.E. Kuil, J.G. Hollander, Self-diffusion and cooperative diffusion of globular proteins in solution, Journal of Physical Chemistry B 103(46) (1999) 10294-10299.

[26] F. Weinbreck, H.S. Rollema, R.H. Tromp, C.G. de Kruif, Diffusivity of whey protein and gum arabic in their coacervates, Langmuir 20(15) (2004) 6389-6395.

[27] J.C. Cardoso, R.L.C. Albuquerque, F.F. Padilha, F.O. Bittencourt, O. de Freitas, P.S. Nunes, N.L. Pereira, M.J.V. Fonseca, A.A.S. Araújo, Effect of the Maillard reaction on properties of casein and casein films, Journal of thermal analysis and calorimetry 104(1) (2011) 249-254.

[28] M.P. Yadav, G.D. Strahan, S. Mukhopadhyay, A.T. Hotchkiss, K.B. Hicks, Formation of corn fiber gum-milk protein conjugates and their molecular characterization, Food hydrocolloids 26(2) (2012) 326-333.

[29] S.M.H. Hosseini, Z. Emam-Djomeh, P. Sabatino, P. Van der Meeren, Nanocomplexes arising from protein-polysaccharide electrostatic interaction as a promising carrier for nutraceutical compounds, Food Hydrocolloids 50 (2015) 16-26.

[30] W. Lertittikul, S. Benjakul, M. Tanaka, Characteristics and antioxidative activity of Maillard reaction products from a porcine plasma protein-glucose model system as influenced by $\mathrm{pH}$, Food Chemistry 100(2) (2007) 669-677.

[31] P. Van der Meeren, M. El-Bakry, N. Neirynck, P. Noppe, Influence of hydrolysed lecithin addition on protein adsorption and heat stability of a sterilised coffee cream simulant, International Dairy Journal 15(12) (2005) 1235-1243.

[32] L. Greenspan, Humidity fixed points of binary saturated aqueous solutions, Journal of Research of the National Bureau of Standards 81(1) (1977) 89-96.

[33] D. Sinnaeve, The Stejskal-Tanner equation generalized for any gradient shape-an overview of most pulse sequences measuring free diffusion, Concepts in Magnetic Resonance Part A 40(2) (2012) 39-65.

[34] J.A. De Vries, F.M. Rombouts, A.G.J. Voragen, W. Pilnik, Enzymic degradation of apple pectins, Carbohydrate Polymers 2(1) (1982) 25-33.

[35] L. Jiménez-Castaño, M. Villamiel, P.J. Martín-Álvarez, A. Olano, R. López-Fandiño, Effect of the dry-heating conditions on the glycosylation of $\beta$-lactoglobulin with dextran through the Maillard reaction, Food Hydrocolloids 19(5) (2005) 831-837.

[36] M. Girard, S.L. Turgeon, S.F. Gauthier, Interbiopolymer complexing between $\beta$-lactoglobulin and low-and high-methylated pectin measured by potentiometric titration and ultrafiltration, Food Hydrocolloids 16(6) (2002) 585-591.

[37] A.P. Imeson, D.A. Ledward, J.R. Mitchell, On the nature of the interaction between some anionic polysaccharides and proteins, Journal of the Science of Food and Agriculture 28(8) (1977) 661-668.

[38] E. Dickinson, Interfacial structure and stability of food emulsions as affected by proteinpolysaccharide interactions, Soft Matter 4(5) (2008) 932-942.

[39] J. Liu, Q. Ru, Y. Ding, Glycation a promising method for food protein modification: physicochemical properties and structure, a review, Food Research International 49(1) (2012) 170-183. 
527 [40] M. Corzo-Martínez, C.C. Sánchez, F.J. Moreno, J.M.R. Patino, M. Villamiel, Interfacial and foaming 528 properties of bovine $\beta$-lactoglobulin: Galactose Maillard conjugates, Food Hydrocolloids 27(2) (2012) 529 438-447.

530 [41] U.S. Schmidt, V. Pietsch, C. Rentschler, T. Kurz, H.-U. Endreß, H. Schuchmann, Influence of the 531 degree of esterification on the emulsifying performance of conjugates formed between whey protein 532 isolate and citrus pectin, Food Hydrocolloids 56 (2016) 1-8.

533 [42] A.D. Setiowati, S. Saeedi, W. Wijaya, P. Van der Meeren, Improved heat stability of whey protein 534 isolate stabilized emulsions via dry heat treatment of WPI and low methoxyl pectin: Effect of pectin concentration, pH, and ionic strength, Food Hydrocolloids 63 (2017) 716-726. [43] Y.-W. Shu, S. Sahara, S. Nakamura, A. Kato, Effects of the length of polysaccharide chains on the functional properties of the Maillard-type lysozyme- polysaccharide conjugate, Journal of Agricultural

539 [44] K. Gu, J. Onorato, S.S. Xiao, C.K. Luscombe, Y.-L. Loo, Determination of the molecular weight of 540 conjugated polymers with diffusion-ordered NMR spectroscopy, Chemistry of Materials 30(3) (2018) $541570-576$. 